\title{
Metastable fragment production by electron-impact dissociation of $\mathrm{SO}_{2}$
}

\author{
P.J.M. van der Burgt, M.E. Antaya, J.W. McConkey \\ Department of Physics, University of Windsor, Windsor, Ontario N9B3P4, Canada
}

Received: 10 December 1991

\begin{abstract}
Time-of-flight detection of neutral metastable fragments has been applied to the study of electron-impact dissociation of $\mathrm{SO}_{2}$ molecules. The time-of-flight distributions indicate that a variety of processes are involved in the production of metastable fragments with a wide range of energies. Rydberg $\mathrm{S}$ and $\mathrm{O}$ atoms and $\mathrm{SO}$ molecules have been identified. Data for these processes have been measured up to $300 \mathrm{eV}$. The results presented are compared with other studies of fragmentation of $\mathrm{SO}_{2}$.
\end{abstract}

PACS: $34.80 . \mathrm{G}$

\section{Introduction}

Interest in electron-impact dissociation processes in $\mathrm{SO}_{2}$ discharges has been stimulated recently by the suggested use of electrical discharges as a means of destruction and removal of this and other pollutant molecules from the exhaust gases of electricity generation stations (Penetrante et al. [21]). Prior to this, considerable interest in $\mathrm{SO}_{2}$ dissociation bas been stimulated by its detection in planetary atmospheres and interstellar clouds. It is a major constituent in the atmosphere of Jupiter's satellite Io. During the Voyager 1 and 2 encounters with Jupiter extreme ultraviolet emissions were observed to occur at a distance of about five times the Jupiter radius, coinciding with the orbit of Io (Broadfoot et al. [6], Sandel et al. [24]). These emissions are attributed to S III, S IV, and O III and it has been argued that the $\mathrm{SO}_{2}$ emerging from volcanic eruptions on the surface of Io is being excited, dissociated and ionized by electron and photon impact, a process that contributes ions to the plasma torus around Jupiter. In addition $\mathrm{SO}_{2}$ is a constituent of the atmosphere of Venus ( $\mathrm{Na}$ et al. [18]). In view of these observations understanding of the dissociation processes that take place under electron impact on $\mathrm{SO}_{2}$ is of great relevance.

Information on dissociation processes comes from a variety of areas of research. Electron impact mass spec- trometry studies have been performed first by Smyth and Muller [26], followed by Reese et al. [22] and Botter et al. [4]. Smith and Stevenson [25], Cadež et al. [8], and Orient and Srivastava [20] have measured partial and total electron-impact ionization cross sections. U1traviolet and visible emissions of $\mathrm{SO}_{2}$ molecules and their fragments have been reported by Becker et al. [3], Miller and Becker [17], Johnson et al. [16] and Ajello et al. $[1,2]$. Dissociative electron attachment has been studied by Orient and Srivastava [19] and Spyrou et al. [28]. Vus̀ković and Trajmar [31] and Trajmar and Shyn [29] have studied differential electron scattering. Photodissociation has been studied by Brehm et al. [5], Dujardin and Leach [11], Dujardin et al. [12], Curtis and Eland [10], Felder et al. [13] and others. The present paper presents the results of a study of dissociative production of metastable neutral fragments by electron impact on $\mathrm{SO}_{2}$.

\section{Experiment}

The apparatus used in these studies is described by van der Burgt and McConkey [30]. Briefly, a pulsed electron beam intersects, at right angles, a gas beam of $\mathrm{SO}_{2}$ molecules effusing from a single hypodermic needle. A metastable detector, comprising of two high-transparency grids followed by a channeltron is mounted at right angles to both the electron and gas beams. The cone of the channeltron is at $100 \mathrm{~mm}$ distance from the intersection of both beams. The grid closest to the channeltron is biased positively and the channeltron cone is biased negatively to prevent the detection of charged particles. The grid closest to the intersection of both beams is kept at ground potential to keep a field-free time-of-flight path.

The electron beam is pulsed by applying a pulse to the Wehnelt cylinder of a width of $0.5 \mu$ s and a repetition rate of $5.6 \mathrm{kHz}$. Faraday cup currents of the order of $50 \mathrm{nA}$ (integrated) are used. The energy of the electron beam is calibrated by examining the threshold excitation 
of the $2^{3} \mathrm{~S}$ metastable state of helium (using an electron pulse repetition rate of $1.6 \mathrm{kHz}$ ). This measurement also indicates that the energy spread in the electron beam is about $0.8 \mathrm{eV}$ FWHM. The effective resolution in the excitation functions measured with $\mathrm{SO}_{2}$ is somewhat less due to the gradual deterioration of the filament in an $\mathrm{SO}_{2}$ environment. Energy calibrations before and after the $\mathrm{SO}_{2}$ measurements indicate a shift of $0.3 \mathrm{eV}$. Without a gas beam the pressure in the vacuum chamber is 1.1 $\times 10^{-7}$ Torr, whereas pressures of about $5 \times 10^{-5}$ Torr are recorded when the $\mathrm{SO}_{2}$ beam is turned on.

Data accumulation is controlled by a fast multichannel scaler described previously (van der Burgt and McConkey [30]). Time-of-flight spectra are recorded by externally triggering the multichannel scaler, synchronously with the electron beam pulse. In this type of measurement dwell times of $200 \mathrm{~ns}$ per channel are used, resulting in a time-of-flight range of $102 \mu \mathrm{s}$. This range is sufficient in studies of $\mathrm{SO}_{2}$ because no significant signal is detected at longer flight times.

All excitation functions are measured simultaneously by computer controlled operation of the multichannel scaler. The Turbo Pascal code controlling the data taking process runs on an XT type personal computer. The computer commands the scaler to accumulate a time-offlight spectrum for a particular electron energy. When the scaler is finished, the accumulated spectrum is transferred to the computer, the ramp voltage for the electron energy is increased, and the scaler contents are cleared for the next time-of-flight spectrum. This process is repeated until a scan across the full range of electron energies is finished and a new scan can be started. In order to reduce the total amount of data the computer only saves and accumulates the relevant part of the time-offlight spectra. In this way all excitation functions are obtained from the same data set. The data set accumulated for $\mathrm{SO}_{2}$ is a two-dimensional array of 20 channels ranging from 9.8 to $29.8 \mu$ s time-of-flight by 250 channels ranging from 16.3 to $78.8 \mathrm{eV}$ electron energy. This method of data collection greatly reduces the total data collection time, a necessity for $\mathrm{SO}_{2}$ due to the limited lifetime of the filament in an $\mathrm{SO}_{2}$ environment. The $\mathrm{SO}_{2}$ data presented here has been collected in 85 hours.

\section{Results}

\subsection{Time-of-flight spectra and kinetic energy distributions}

Time-of-flight spectra for five different electron energies are shown in Fig. 1. All spectra have been taken using electron pulses of $0.5 \mu$ s width and a repetition rate of $5.6 \mathrm{kHz}$. The origin of the time-of-flight scale is determined by a prompt peak produced by radiative decay of excited fragments. Fragments decaying in their flight towards the detector produce a trailing edge on the prompt peak (not shown in Fig. 1).

Kinetic energy distributions are obtained by using the transformation given by (3) and (4) of Smyth et al. [27] and are shown in Fig. 2. The distributions shown cover a range from 8.3 to $75 \mu$ s. As the transformation

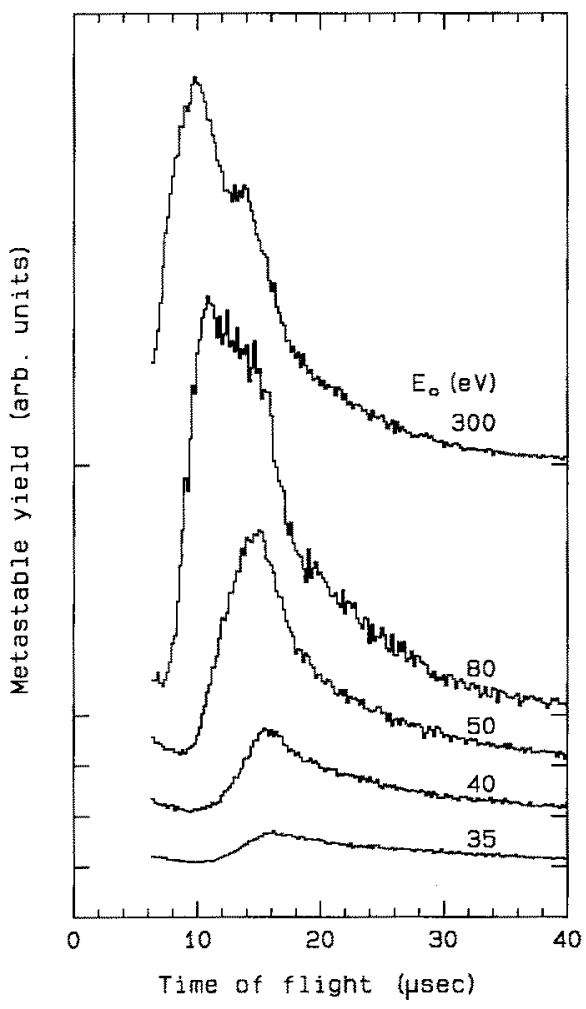

Fig. 1. Time-of-flight spectra for metastable fragments obtained at the incident electron energies indicated. Vertical scales are comparable to within $20 \%$

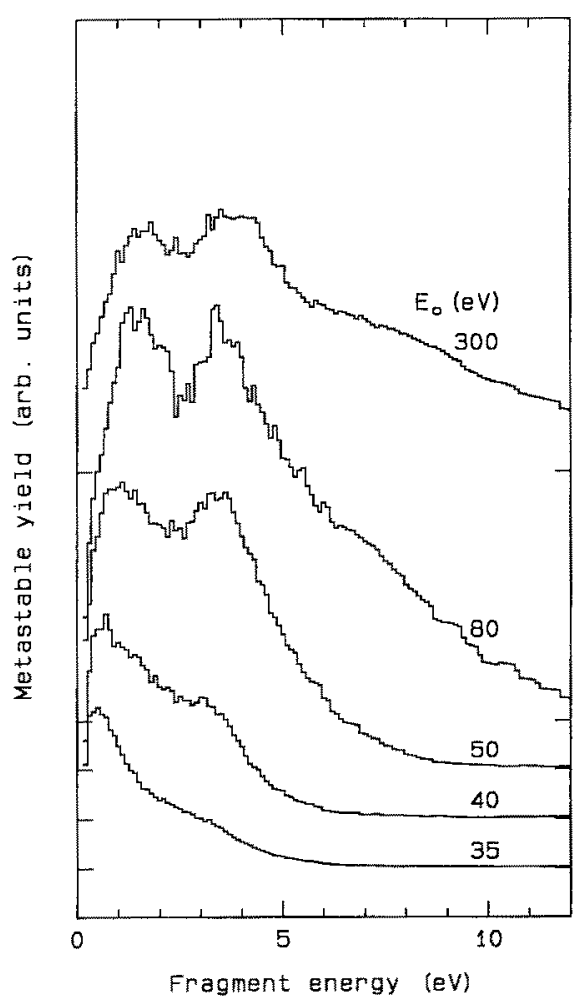

Fig. 2. Kinetic energy spectra obtained from the spectra in Fig. 1, assuming the detected metastable fragment is oxygen. The spectra cover a range from 8.3 to $75 \mu$ s and the time-of-flight path is $100 \mathrm{~mm}$ 
involves a factor $t^{3}$ a large number of data points are transformed into a narrow region of small fragment energies. In order to avoid the clutter of points in this region the transformation is performed numerically using equal energy steps of $0.1 \mathrm{eV}$. Because the detector pulses are delayed $4.8 \mu \mathrm{s}$, the first 24 channels in the time-of-flight spectra correspond to the longest flight times possible following the preceding electron pulse. The average number of counts in these channels is used as the baseline level in the transformation to kinetic energy.

The kinetic energy distributions must be interpreted with some care, because the transformation assumes that atomic oxygen fragments are detected. Contributions could be present due to metastable $\mathrm{S}, \mathrm{SO}$ or $\mathrm{O}_{2}$ fragments and, as discussed later, all of these species are in fact present. The metastable detector is able to detect those metastable species with excitation energies greater than the work function of the channeltron (estimated to be about $8 \mathrm{eV}$ by Brunt et al. [7]). Consequently, only $\mathrm{O}\left(3 s^{5} S^{0}\right)$ metastable atoms at $9.14 \mathrm{eV}$ are detected, whereas $\mathrm{O}\left(2 p^{41} D\right), \mathrm{O}\left(2 p^{41} S\right), \mathrm{S}\left(3 p^{41} D\right), \mathrm{S}\left(3 p^{41} S\right)$, and $S\left(4 s^{5} S^{0}\right)$ metastable atoms do not produce any signal. Oxygen and sulphur atoms in long-lived Rydberg states can be detected as well.

It is clear from Figs. 2 and 3 that three groups of fragments are represented, at short, medium and long flight times (high, medium and low energies). These three groups may be further subdivided as discussed below.

\subsection{Appearance potentials and excitation functions}

As described above, 20 excitation functions have been measured for $1 \mu \mathrm{s}$ time-of-flight windows ranging from 9.8 to $29.8 \mu \mathrm{s}$. Four excitation functions are displayed in Fig. 3. Onsets are identified in this figure. Because a number of different dissociation processes are contrib-

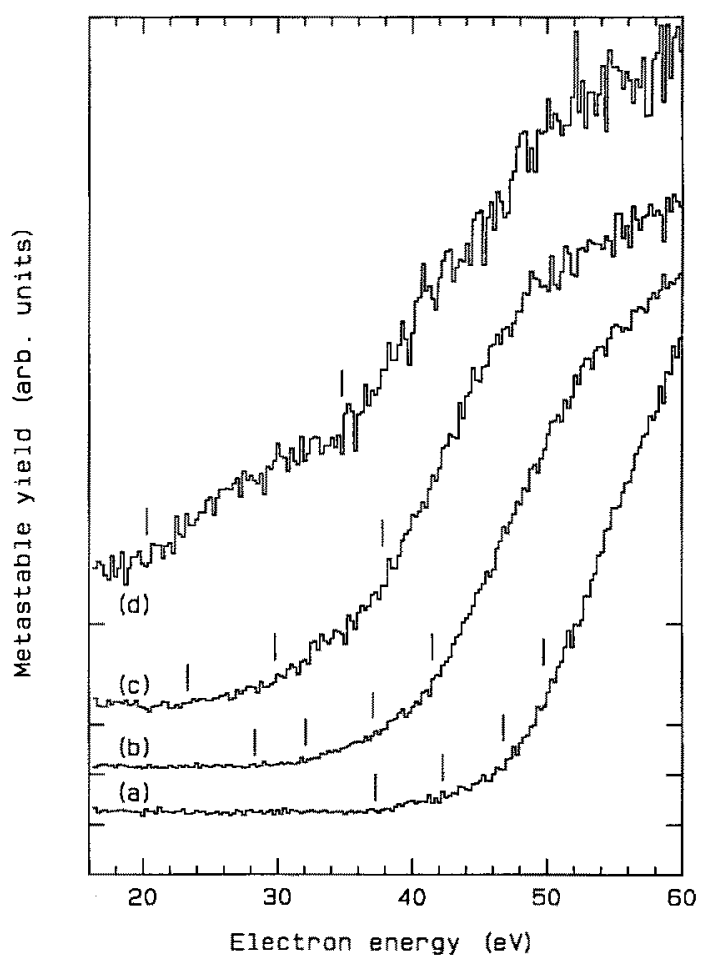

Fig. 3. Excitation functions measured for different fragment kinetic energies: $6.0-7.1 \mathrm{eV}(a), 3.8-4.4 \mathrm{eV}(b), 2.11-2.35 \mathrm{eV}(c)$, and $0.93-$ $1.00 \mathrm{eV}(d)$. Marks indicate the onsets of dissociation processes. Vertical scales are not comparable

uting to the yield of metastables, the excitation functions exhibit sharp increases in slope at one or more energies corresponding to the thresholds of the contributing dissociation processes.

These onsets have been located by drawing straight line segments (or curved line segments with decreasing slope) in the graphs (not shown in Fig. 3). The identifica-

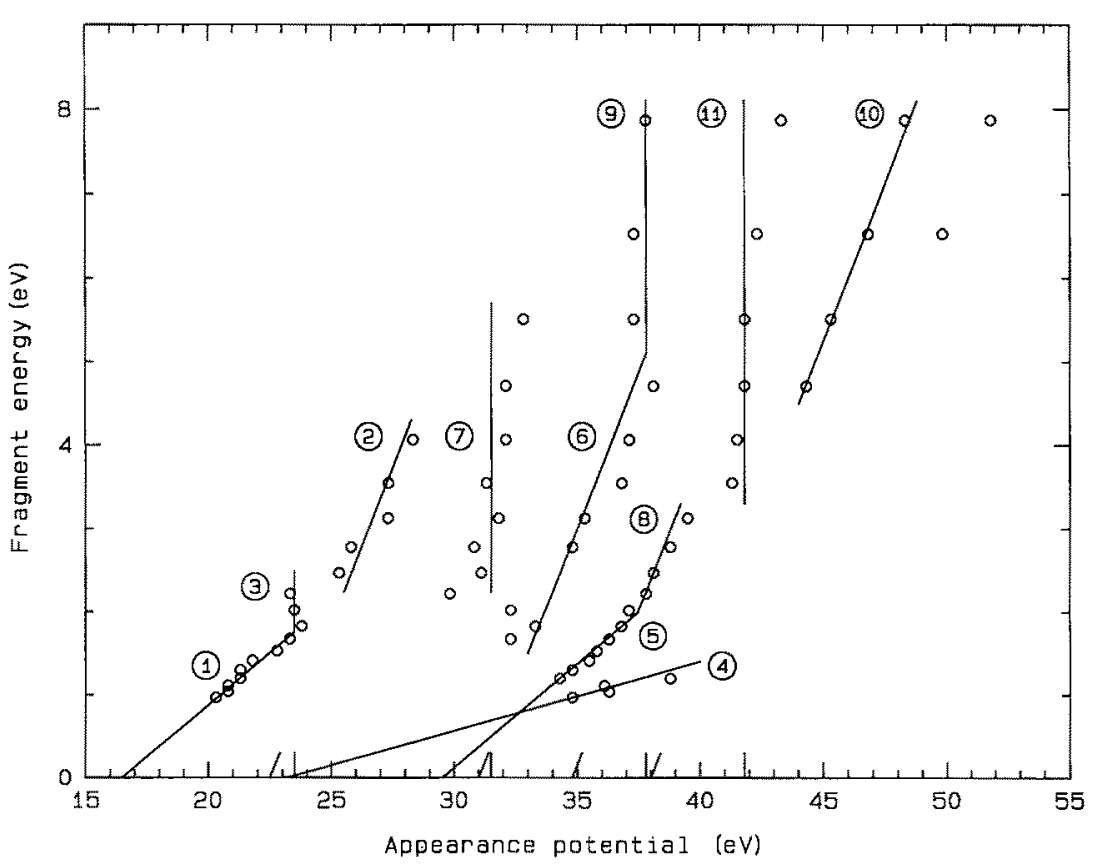

Fig. 4. Plot of appearance potentials against fragment kinetic energies, assuming the detected metastable fragment is $O$. The lines with slope 0.75 are due to two-fragment dissociations producing metastable oxygen atoms and the vertical lines indicate multiple fragment dissociations (see text). Lines with slope 0.25 are due to metastable sulphur (or metastable $\mathrm{O}_{2}$ ). The line with slope 0.083 is due to metastable SO 
tion of onsets is often difficult and in many cases only possible by extending the vertical scale of the graph. Even in data with good statistics an onset at a higher energy results in a small increase superimposed on a large signal from lower thresholds, and is only indicated by a minor inflection in the accumulated counts. We estimate the error in the onset energies to be about $0.5 \mathrm{eV}$ for well defined onsets, and about $1 \mathrm{eV}$ or more for onsets in the threshold region (poor statistics) and onsets indicated by a minor inflection.

A plot of fragment kinetic energies against appearance potentials (location of onsets on a calibrated energy scale) is shown in Fig. 4. Assuming an oxygen atom is detected as a result of a two-fragment break-up (and no internal energy is carried off in the other fragment) the slope $\alpha$ of appearance potentials versus fragment energies follows from conservation of momentum:

$\alpha=m(\mathrm{SO}) / m\left(\mathrm{SO}_{2}\right)=0.75$

In case of a two-fragment break-up with detection of $\mathrm{S}$ or $\mathrm{O}_{2}$ a slope of 0.25 is obtained in plots such as in Fig. 4. Detection of SO results in a slope of 0.083 . Since $\mathrm{SO}_{2}$ in its ground configuration is bent with an enclosed angle of $120^{\circ}$, symmetric break up, with ejection of two oxygen atoms, would be evidenced by a slope of 0.4 .

For dissociation of polyatomic molecules, molecular fragments with different amounts or rotational and vibrational excitation might be produced, or multiple fragmentation may occur. These situations complicate the relation between appearance potentials and fragment energies (see the review by Freund [14]). In the first situation, if a constant fraction of the excess energy is converted into translational motion, again a constant slope is obtained. In the second situation, two or more undetected fragments are produced with varying kinetic energy, and a vertical slope is obtained. Figure 4 shows that some series of appearance potentials follow straight lines that closely match the predicted slope for a two-fragment break-up producing metastable oxygen atoms. Series of appearance potentials with vertical slope indicate that multiple fragmentation processes also occur. Two series of appearance potentials indicate two fragment break-up with detection of either metastable $\mathrm{S}$ or $\mathrm{O}_{2}$, and one set of appearance potentials is possibly due to production of metastable SO.

\section{Discussion of fragmentation processes}

Figure 4 indicates that a variety of dissociation processes is responsible for the formation of metastable fragments. Possible processes indicated in Fig. 4 have been labelled 1 to 11 in order of increasing dissociation limit, (indicated by extrapolated intercept with the energy axis). Definitive identification of the processes involved is often difficult in view of the great many possible channels. Literature values for dissociation and ionization energies are used to enable identification of these processes. Ionization energies for various molecular fragments are tabu- lated by Rosenstock et al. [23], and dissociation energies can be obtained from enthalpies of formation listed by Chase et al. [9]. Oxygen and sulphur Rydberg atoms (denoted by $O(R)$ and $S(R)$ in the equations below) have excitation energies close to their ionization energies of $13.61 \mathrm{eV}$ and $10.36 \mathrm{eV}$, respectively, and oxygen Rydberg molecules $\left(\mathrm{O}_{2}(\mathrm{R})\right)$ have excitation energies close to $12.07 \mathrm{eV} . \mathrm{O}\left(3 \mathrm{~s}^{5} S^{0}\right)$ has an excitation energy of $9.14 \mathrm{eV}$.

Four series of onsets in Fig. 4, (labelled processes \#2, 6,8 and 10) have slopes which indicate that two-fragment break-ups producing metastable oxygen atoms with dissociation limits at 22.5, 31.0, 34.8 and $38.0 \mathrm{eV}$ are occuring.

\section{Process \# 2 (limit $22.5 \mathrm{eV})$}

Using the dissociation energy (Chase et al. [9]) of $5.66 \mathrm{eV}$ for break up of $\mathrm{SO}_{2}$ into $\mathrm{SO}+\mathrm{O}$ together with the excitation energies of SO (Huber and Herzberg [15]), we find the following possible assignments for this process

$$
\begin{aligned}
& e+\mathrm{SO}_{2} \rightarrow \mathrm{SO}\left(X^{3} \Sigma^{-}\right)+\mathrm{O}(\mathrm{R})+e^{\prime} \\
& e+\mathrm{SO}_{2} \rightarrow \mathrm{SO}^{*}+\mathrm{O}\left(3 s^{5} \mathrm{~S}^{0}\right)+e^{\prime}
\end{aligned}
$$

with calculated limits of $19.3 \mathrm{eV}$ (2) and $19.6 \mathrm{eV}$ or greater (3) depending on whether the $\mathrm{SO}^{*}$ fragment is left in the $A^{3} \Pi$ or a higher state. The small difference in energies between the observed and calculated limits could readily be explained in terms of some vibrational excitation of the molecular fragment. $\mathrm{SO}\left(A^{3} \Pi\right)$ has been observed to be strongly excited following electron impact on $\mathrm{SO}_{2}$ (Ajello et al. [2] and Miller and Becker [7]). However, (2) might be favoured because of its less severe requirements for spin conservation. In addition, mass spectrometer studies by Reese et al. [22] and Orient and Srivastava [20] report appearance potentials for the production of $\mathrm{O}^{+}$at 20.6 and $23.5 \mathrm{eV}$, respectively. These appearance potentials have been attributed to fragmentation into $\mathrm{SO}+\mathrm{O}^{+}$. They compare well with our measured dissociation limit of $22.5 \mathrm{eV}$ for $\mathrm{O}(\mathrm{R})$ production by reaction (2).

\section{Process \#6 (limit $31.0 \mathrm{eV})$}

Using the dissociation energy of $\mathrm{SO}_{2}$ as before together with the ionization energy of SO $(10.35 \mathrm{eV}$, Rosenstock et al. [23]) we find the most likely assignment of this process as

$e+\mathrm{SO}_{2} \rightarrow \mathrm{SO}^{+}\left(X^{2} \Pi\right)+\mathrm{O}(\mathrm{R})+2 e$

with a calculated dissociation limit of $29.6 \mathrm{eV}$. Again some vibrational excitation of the molecular fragment might be occurring.

Processes \#8 and \#10 (limits 34.8 and $38.0 \mathrm{eV}$ )

Here it seems likely that reactions, similar to (4) are occurring but with the $\mathrm{SO}^{+}$ion being left in an excited 
state. Thus if $\mathrm{SO}^{+}$is produced in the $A^{2} \Pi, B^{2} \Sigma$ or $C^{2} \Pi$ states the dissociation limits would be $34.0,35.8$ and $39.2 \mathrm{eV}$, respectively. By observing double photoionization using a photoion-photoion coincidence technique, Dujardin et a1. [12] find onsets for the production of $\mathrm{SO}^{+}+\mathrm{O}^{+}$at $34.1 \mathrm{eV}$ (with $4.7 \mathrm{eV}$ total kinetic energy) and $41 \mathrm{eV}$ (with $7 \mathrm{eV}$ total kinetic energy). They attribute these onsets to dissociations producing ground state $\mathrm{SO}^{+}$and excited $\mathrm{SO}^{+}\left(A^{2} \Pi\right.$ or $\left.b^{4} \Sigma^{-}\right)$, respectively. These observations compare with our $\mathrm{SO}^{+}+\mathrm{O}(\mathrm{R})$ dissociation limits at 31.0 and $34.8 \mathrm{eV}$.

A further possibility which should be mentioned in connection with process \# 8 is that of symmetric breakup with ejection of two O-atom fragments. As mentioned earlier, this would lead to onsets lying on a line of slope 0.4 . Such a line could be drawn through the onsets identified with process $\# 8$ in Fig. 4 with an extrapolated threshold at $32.0 \mathrm{eV}$.

Two series of onsets labelled 1 and 5 in Fig. 4 lie on lines of slope 0.25 with limits at 16.5 and $29.5 \mathrm{eV}$. These indicate two-particle fragmentation into Rydberg $\mathrm{S}$ or $\mathrm{O}_{2}$ fragments.

\section{Process \#1 (limit $16.5 \mathrm{eV})$}

Making similar energy calculations as before strongly suggests that this process is

$e+\mathrm{SO}_{2} \rightarrow \mathrm{S}(\mathrm{R})+\mathrm{O}_{2}\left(X^{3} \Sigma_{g}^{-}\right)+e^{\prime}$

with a calculated limit of $16.0 \mathrm{eV}$. Production of $\mathrm{O}_{2}(\mathrm{R})$ with a sulphur atom is not energetically feasible at this energy.

Support for the assignment given in (5) comes from the mass spectrometer studies of Reese et al. [22] and Orient and Srivastava [20]. They report an appearance potential for a mass 32 fragment at 17.5 and $16.5 \mathrm{eV}$, respectively. The yield of ions must be due to $\mathrm{S}^{+}$because both groups report a small signal at mass 34 , in correspondence with the naturally occurring S-isotopes.

Process \#5 (limit $29.5 \mathrm{eV}$ )

Two possible reactions for this process are

$e+\mathrm{SO}_{2} \rightarrow \mathrm{S}(\mathrm{R})+\mathrm{O}_{2}^{+}\left(X^{2} \Pi_{\mathrm{g}}\right)+2 e$

$e+\mathrm{SO}_{2} \rightarrow \mathrm{S}^{+}\left(3 p^{3}{ }^{4} \mathrm{~S}^{0}\right)+\mathrm{O}_{2}(\mathrm{R})+2 e$

Both (6) and (7) have a calculated limit of $28.3 \mathrm{eV}$ in good agreement with our measured value for process 5 .

\section{Process \# 4 (limit $23.2 \mathrm{eV}$ )}

One series of four onsets with slope 0.083 seems to clearly indicate a two fragment process yielding metastable SO. Fragmentation into $\mathrm{SO}(\mathrm{R})+\mathrm{O}$ has a calculated limit of $16.0 \mathrm{eV}$ if both fragments are unexcited and so it seems likely that the process is

$e+\mathrm{SO}_{2} \rightarrow \mathrm{SO}(\mathrm{R})+\mathrm{O}^{*}+e^{\prime}$ with calculated limits of $20.2 \mathrm{eV}$ if the excited O-atom is in the $2 p^{4} S$ state or $25.5 \mathrm{eV}$ if it is in one of the higher excited states, for example $3 s^{3} S^{0}$. Unfortunately, fragments with energies less than $1 \mathrm{eV}$ were not studied in this experiment and so the extrapolated limit of process \# 4 has somewhat more uncertainty in its position than some of the other limits. We note that $3 s^{3} S^{0}$ has been observed to be strongly excited in other electronimpact studies (Ajello et al. [1]). These authors observe an onset in their excitation function of the $\mathrm{OI}\left(3 s^{3} S^{0}\right.$ $-3 p^{3} P$ ) $130.3 \mathrm{~nm}$ line at $24 \mathrm{eV}$ close to our measured onset for process \#4. Further support for the identification of this process with $\mathrm{SO}(\mathrm{R})$ fragments comes from the mass spectrometry work (Orient and Srivastava [20], Reese et al. [22], Smith and Stevenson [25]). All of these authors observe $\mathrm{SO}^{+}$as the dominant fragment ion and thus $\mathrm{SO}(\mathrm{R})$ based on this ion core would also be expected to be a strong feature.

Processes \#3, 7, 9 and 11 (limits 23.5, 31.5, 37.8 and $41.8 \mathrm{eV})$

Four vertical series of onsets at the energies listed above indicate total fragmentation of the $\mathrm{SO}_{2}$ molecule. As any of the atoms may end up in a large number of possible excited states an equally large number of possible assignments exist for these thresholds. For example the onset at $23.5 \mathrm{eV}$ (process \#3) may be due to

$e+\mathrm{SO}_{2} \rightarrow \mathrm{S}(\mathrm{R})+\mathrm{O}\left(2 p^{43} P\right)+\mathrm{O}\left(2 p^{43} P\right)+e^{\prime}$

with a calculated limit of $21.5 \mathrm{eV}$ or to

$e+\mathrm{SO}_{2} \rightarrow \mathrm{S}(\mathrm{R})+\mathrm{O}\left(2 p^{41} D\right)+\mathrm{O}\left(2 p^{43} P\right)+e^{\prime}$
$e+\mathrm{SO}_{2} \rightarrow \mathrm{S}\left(3 p^{43} P\right)+\mathrm{O}(\mathrm{R})+\mathrm{O}\left(2 p^{43} P\right)+e^{\prime}$

with calculated limits of 23.4 and $24.7 \mathrm{eV}$, respectively. If, as seems possible from Fig. 4, processes \#1 and \#3 are linked together then (9) might be favoured, as this process is equivalent to process \#1, (5), if additional dissociation of the $\mathrm{O}_{2}$ molecule occurs. Support for this identification comes from the work of Ajello et al. [1] who observe a strong threshold at $19.5 \mathrm{eV}$ in the excitation function of the $\operatorname{SI}\left(4 s^{\prime 3} D^{0}-3 p^{4} P\right) 147.8 \mathrm{~nm}$ line which they attribute to total fragmentation of the parent molecule. Also mass spectroscopy studies provide an indication for reaction (9). Reese et al. [22] report a second onset at $22.6 \mathrm{eV}$ (appearance potential at $17.5 \mathrm{eV}$ ) whereas Smith and Stevenson [25] report an appearance potential at $22 \mathrm{eV}$ for mass 32 . These onsets have been attributed to a fragmentation into $\mathrm{S}^{+}+20$.

For the higher limits at $31.5,37.8$ and $41.8 \mathrm{eV}$ we mention the possibilities

$e+\mathrm{SO}_{2} \rightarrow \mathrm{S}(\mathrm{R})+\mathrm{O}^{+}\left(2 p^{34} S^{0}\right)+\mathrm{O}\left(2 p^{43} P\right)+2 e$

with a calculated limit of $31.2 \mathrm{eV}$

$e+\mathrm{SO}_{2} \rightarrow \mathrm{S}\left(2 p^{43} P\right)+\mathrm{O}(\mathrm{R})+\mathrm{O}^{+}\left(2 p^{34} S^{0}\right)+2 e$ 
with a calculated limit of $38.2 \mathrm{eV}$ and

$e+\mathrm{SO}_{2} \rightarrow \mathrm{S}\left(3 p^{43} P\right)+\mathrm{O}(\mathrm{R})+\mathrm{O}^{+}\left(2 p^{32} D^{0}\right)+2 e$

with a calculated limit of $41.6 \mathrm{eV}$. However, because of the multiple assignments which exist for these limits it is impossible to be definitive in these cases.

As discussed in Sect. 3.2, the determination of onsets is sometimes a difficult task. In the present work the authors determined onsets independently and found good agreement for most of the onsets. However, some ambiguity remains in the $27-34 \mathrm{eV}$ region of the excitation functions around $2 \mathrm{eV}$ fragment kinetic energy and in the onsets of the excitation functions above $6 \mathrm{eV}$ fragment kinetic energy.

\section{Conclusions}

We have studied the production of metastable fragments by electron impact dissociation of $\mathrm{SO}_{2}$ molecules. The results show that a variety of different processes contribute to the formation of metastable fragments and that very energetic fragments up to $20 \mathrm{eV}$ can be produced. Our results indicate the importance of both two and three fragment dissociation processes producing excited oxygen and sulphur atoms and SO molecules and molecular ions.

The authors gratefully acknowledge financial support from the Natural Sciences and Engineering Rescarch Council of Canada

\section{References}

1. Ajello, J.M., James, G.K., Kanik, I., Franklin, B.O.: submitted to J. Geophys. Res. (1991 a)

2. Ajello, J.M., James, G.K., Kanik, I., Shemansky, D.E.: submitted to J. Geophys. Res. (1991 b)

3. Becker, K., Wijngaarden, W. van, McConkey, J.W.: Planet. Space Sci. 31, 197 (1983)

4. Botter, R., Hagemann, R., Nief, G., Roth, F.: Adv. Mass Spectr., vol. 3, p. 951. London: Institute of Petroleum (1960)

5. Brehm, B., Eland, J.H.D., Frey, R., Künstler, A.: Int. J. Mass Spectr. Ion Phys, 12, 197 (1973)

6. Broadfoot, A.L., Belton, M.J.S., Takacs, P.Z., Sandel, B.R., Shemansky, D.E., Holberg, J.B., Ajello, J.M., Atreya, S.K., Don- ahue, T.M., Moos, H.W., Bertaux, J.L., Blamont, J.E., Strobel, D.F., McConnell, J.C., Dalgarno, A., Goody, R., McElroy, M.B.: Science 204, 979 (1979)

7. Brunt, J.N.H., King, G.C., Read, F.H.: J. Phys. B. 11, 173 (1978)

8. Cadež, I.M., Pejčev, V.M., Kurepa, M.V.: J. Phys. D. 16, 305 (1983)

9. Chase, M.W. Jr., Davies, C.A., Downey, J.R. Jr., Frurip, D.J., McDonald, R.A., Syverud, A.N.: J. Phys. Chem. Ref. Data 14, Suppl. 1 (JANAF Thermochemical Tables, 3rd ed.) (1985)

10. Curtis, D.M., Eland, J.H.D.: Int. J. Mass Spectr. Ion Proc. 63, $241(1985)$

11. Dujardin, G., Leach, S.: J. Chem. Phys. 75, 2521 (1981)

12. Dujardin, G., Leach, S., Dutuit, O., Guyon, P.-M., RichardViard, M.: Chem. Phys. 88, 339 (1984)

13. Felder, P., Effenhauser, C.S., Haas, B.M., Huber, J.R.: Chem. Phys. Lett. 148, 417 (1988)

14. Freund, R.S.: In: Rydberg states of atoms and molecules, p. 355. Stebbings, R.F.s Dunning, F.B. (eds). Cambridge: Cambridge University Press 1983

15. Huber, K.P., Herzberg, G.: Molecular spectra and molecular structure IV. Constants of diatomic molecules. New York: Van Nostrand Reinhold 1979

16. Johnson, C.A.F., Kelly, S.D., Park, J.E.: J. Chem. Soc. Faraday Trans. 2 83, 411 and 985 (1987)

17. Miller, K. Jr., and Becker, K.: Can. J. Phys. 65, 530 (1987)

18. Na, C.Y., Esposito, L.W., Skinner, T.E.: J. Geophys. Res. 95, $7485(1990)$

19. Orient, O.J., Srivastava, S.K.: J. Chem. Phys. 78, 2949 (1983)

20. Orient, O.J., Srivastava, S.K.: J. Chem. Phys. 80, 140 (1984)

21. Penetrante, B.M., Bardsley, J.N., Vitello, P.A., Vogtlin, G.E., Hofer, W.W.: 44th gaseous electronics conference, Albuquerque, New Mexico, Abstracts, p. 159 (1991)

22. Reese, R.M., Dibeler, V.H., Franklin, J.I.: J. Chem. Phys. 29, $880(1958)$

23. Rosenstock, H.M., Draxl K., Steiner, B.W., Herron, J.T.: J. Phys. Chem. Ref. Data 6, Suppl. 1 (1977)

24. Sandel, B.R., Shemansky, D.E., Broadfoot, A.L., Bertraux, J.L., Blamont, J.E., Belton, M.J.S., Ajello, J.M., Holberg, J.B., Atreya, S.K., Donahue, T.M., Moos, H.W., Strobel, D.F., McConnell, J.C., Dalgarno, A., Goody, R., McElroy, M.B., Takacs, P.Z.; Science 206, 962 (1979)

25. Smith, O.I., Stevenson, J.S.: J. Chem. Phys. 74, 6777 (1981)

26. Smyth, H.D., Muller, D.W.: Phys. Rev. 43, 121 (1933)

27. Smyth, K.C., Schiavone, J.A., Freund, R.S. I. Chem. Phys. 59, $5225(1973)$

28. Spyrou, S.M., Sauers, I., Christophorou, L.G.: J. Chem. Phys. 84, 239 (1986)

29. Trajmar, S., Shyn, T.W.: J. Phys. B 22, 2911 (1989)

30. Burgt, P.J.M. van der, McConkey, J.W.: J. Phys. B 24, 4821 (1991)

31. Vus̀ković, L., Trajmar S.: J. Chem. Phys. 77, 5436 (1982) 